\title{
A single serving of caffeinated coffee impairs postprandial glucose metabolism in overweight men
}

\author{
Tracey M. Robertson ${ }^{1}$, Michael N. Clifford ${ }^{1}$, Simon Penson ${ }^{2}$, Gemma Chope $^{2}$ and M. Denise Robertson ${ }^{1 *}$ \\ ${ }^{1}$ Department of Nutritional Sciences, University of Surrey, Guildford GU2 7WG, UK \\ ${ }^{2}$ Department of Primary Production and Processing, Campden BRI, Chipping Campden GL55 6LD, UK
}

(Submitted 2 February 2015 - Final revision received 2 June 2015 - Accepted 18 June 2015 - First published online 24 August 2015)

\section{Abstract}

Previous studies regarding the acute effects of coffee on glycaemic control have used a single large dose of coffee, typically containing the caffeine equivalent of 2-4 servings of coffee. This study investigates whether the acute effects of coffee are dose-dependent, starting with a single serving. A total of ten healthy overweight males participated in a two-part randomised double-blind cross-over study. In the first part, they ingested 2, 4 or $8 \mathrm{~g}$ instant decaffeinated coffee (DC) dissolved in $400 \mathrm{ml}$ water with caffeine added in proportion to the DC (total 100,200 or $400 \mathrm{mg}$ caffeine) or control ( $400 \mathrm{ml}$ water) all with $50 \mathrm{~g}$ glucose. In the second part, they ingested the same amounts of DC (2, $4,8 \mathrm{~g})$ or control, but with a standard $100 \mathrm{mg}$ caffeine added to each. Capillary blood samples were taken every $15 \mathrm{~min}$ for $2 \mathrm{~h}$ after each drink and glucose and insulin levels were measured. Repeated measures ANOVA on glucose results found an effect when caffeine was varied in line with DC $(P=0.008)$. Post hoc analysis revealed that both 2 and $4 \mathrm{~g}$ DC with varied caffeine content increased the glycaemic response $v$. control. There was no effect of escalating doses of DC when caffeine remained constant at $100 \mathrm{mg}$. These results demonstrate that one standard serving of coffee ( $2 \mathrm{~g}$ ) is sufficient to affect glucose metabolism. Furthermore, the amount of caffeine found in one serving (100 mg) is sufficient to mask any potential beneficial effects of increasing other components. No dose-dependent effect was found.

Key words: Human: Coffee: Caffeine: Glucose: Insulin: Metabolism: Postprandial metabolism

According to the WHO, nearly 350 million people worldwide have diabetes, of whom approximately $90 \%$ have type 2 diabetes mellitus $(\mathrm{T} 2 \mathrm{DM})^{(1)}$. If poorly controlled, diabetes can result in serious complications such as CVD, nephropathy and retinopathy. Much epidemiological evidence suggests that people who drink coffee have a reduced risk of developing $\mathrm{T}_{2} \mathrm{DM}^{(2-5)}$. This has been demonstrated for both caffeinated and decaffeinated coffee (DC) ${ }^{(6,7)}$ and appears to be particularly strong for those who drink large quantities of coffee ${ }^{(8,9)}$; however it is not known precisely how coffee may exert this protective effect. Metabolic syndrome is a group of risk factors that are linked with the development of T2DM. Potential mechanisms where coffee drinking could reduce the risk of T2DM may be through its demonstrated effects on some of these factors. These include aiding weight loss by increasing energy expenditure and fat oxidation ${ }^{(10-12)}$ and reducing energy intake ${ }^{(13)}$, increasing HDL-cholesterol ${ }^{(14)}$ and increasing circulating adiponectin levels ${ }^{(15,16)}$. Adiponectin levels have been shown to be inversely associated with insulin resistance ${ }^{(17)}$; therefore, an increase in levels may result in improved insulin sensitivity, although this has not been found previously, perhaps due to short study durations.

Acute studies about the effects of coffee or caffeine on the postprandial glycaemic response have, however, generally shown a detrimental effect. Greater glucose and insulin AUC have been demonstrated following caffeine ingestion ${ }^{(18,19)}$. Caffeinated coffee ingestion has also produced increased glucose responses compared with a control ${ }^{(20-22)}$; however, the effect appears to be less pronounced than that produced by caffeine alone ${ }^{(23)}$. It has been suggested that bioactive components in coffee, other than caffeine, may be attenuating the documented acute negative effects of caffeine on glycaemia. Chlorogenic acids (CGA) and quinides have attracted attention as possible candidates with suggested mechanisms including delayed glucose absorption by the enterocyte ${ }^{(24)}$ via dissipation of the $\mathrm{Na}^{+}$gradient and consequent inhibition of the SGLT1 glucose transporter ${ }^{(25)}$ in addition to increased uptake of glucose in non-skeletal muscles ${ }^{(26)}$. Studies involving CGA on its Own $^{(27)}$ and coffee enriched with CGA ${ }^{(28)}$ have both demonstrated a reduction in glucose response $v$. control. The varying results from coffee studies may be partially explained by the

Abbreviations: CGA, chlorogenic acids; CQA, caffeoylquinic acid; DC, decaffeinated coffee; iAUC, incremental AUC; T2DM, type 2 diabetes mellitus.

* Corresponding author: Dr M. D. Robertson, fax +44 1483 688501, email m.robertson@surrey.ac.uk 
CGA content of the coffees used, as this can vary considerably ${ }^{(29)}$. However, this cannot be verified as the majority of these studies did not measure CGA content. A limitation to previously published work is the relatively high dose of caffeine used, typically 3-6 mg caffeine/kg body weight ${ }^{(19,30)}$, which equates to $210-420 \mathrm{mg}$ for a $70 \mathrm{~kg}$ person. A Canadian study ${ }^{(31)}$ that analysed the caffeine content of various instant coffees, prepared to participants' personal taste preferences, found a median caffeine concentration in the prepared coffees of $328 \mu \mathrm{g} / \mathrm{ml}$ (range $102-559 \mu \mathrm{g} / \mathrm{ml}$ ). This median value would provide $85 \mathrm{mg}$ caffeine in a standard-sized $(260 \mathrm{ml})$ serving. Doses of $3-6 \mathrm{mg} / \mathrm{kg}$ would, therefore, potentially be equivalent to $2 \cdot 5-5$ standard-sized servings of instant coffee taken as a single dose for a $70 \mathrm{~kg}$ person and correspondingly higher for heavier individuals.

To our knowledge, there has been no study thus far investigating the glycaemic effects of a single serving of instant coffee as consumed in the UK. Instant coffee was chosen for this study because (i) it is the most commonly consumed type of coffee in the $\mathrm{UK}^{(32)}$ and (ii) we wanted to ensure that the levels of the different coffee components were consistent between brews as other types of coffee have been shown to vary in their caffeine and CGA content ${ }^{(33,34)}$. In this pilot investigation, we aimed to perform a dose-response study in overweight individuals with the lowest dose set at a single serving of caffeinated coffee. In Part B of the study, CGA and other coffee components were increased step-wise, whereas the caffeine dose remained constant, to investigate the acute effects of fortification with these other coffee components.

\section{Methods \\ Participants}

Ten participants were recruited from the staff and student population of the University of Surrey. All of them were overweight $\left(\mathrm{BMI}>25 \mathrm{~kg} / \mathrm{m}^{2}\right)$ but otherwise healthy adult males aged 19-62 years who were regular coffee drinkers (>4 servings/week). All of them were non-smokers. Participants were required to have been weight stable for the previous 3 months and to have no history of heart disease, diabetes, liver disease or any gastrointestinal (GI) or endocrine disorders. This study was conducted according to the guidelines laid down in the Declaration of Helsinki, and all procedures involving human subjects were given a favourable ethical opinion by the University of Surrey Ethics Committee. Written informed consent was obtained from all the participants. The study was carried out between August 2012 and February 2013

\section{Study design}

A randomised cross-over design was used to investigate the glycaemic effect of three different doses of DC, to which different doses of caffeine had been added, $v$. a control drink (water). All drinks were made up in $400 \mathrm{ml}$ of water and each had $50 \mathrm{~g}$ glucose powder added. The different drink combinations along with their caffeoylquinic acid (CQA) contents are summarised in Table 1. All treatments, apart from control, were double-blinded. Participants were informed that they were testing different coffee drinks only; they were not told that they contained differing amounts of caffeine and coffee. DC, to which caffeine powder was added, was used so that the effects of caffeine and the other coffee components could be examined separately. The instant coffee granules were a commercially available DC, Kenco Decaff (The Kenco Coffee Company).

The study was split into two parts. Part A looked to establish the effect of different doses of caffeinated coffee on postprandial blood glucose and insulin levels. To achieve this, caffeine was varied in line with the DC dose. Three different doses of DC were used (2, 4 and $8 \mathrm{~g}$ instant granules, equivalent to that found in 1,2 and 4 servings of coffee) to which appropriate doses of caffeine were added to reflect the amount of caffeine found in the same doses of regular caffeinated coffee. As the DC was reported as containing $0 \cdot 3 \%$ caffeine, the amount of caffeine added was adjusted so that the total caffeine in each drink was 100, 200 and $400 \mathrm{mg}$, respectively. These three coffee drinks were compared with the control drink, which contained no caffeine in Part A. Part B looked to establish the effect of escalating doses of the non-caffeine coffee components on postprandial blood glucose and insulin levels by comparing the same three doses of DC as used in Part A, but this time all taken along with the lowest dose of caffeine from Part A that was shown to produce an effect on the postprandial glycaemic response $(100 \mathrm{mg})$. For Part B, the control drink also contained $100 \mathrm{mg}$ caffeine. The relative proportions for each drink are shown in Fig. 1.

\section{Test protocol}

All the participants received each of the eight test beverages in a random order on separate study mornings separated by a washout period of at least $5 \mathrm{~d}$. An online randomisation programme was used to generate the sequences for each participant. For $2 \mathrm{~d}$ before each of the study days, the participants were asked to refrain from exercise, alcohol and all coffee- and caffeine-containing foods and drinks. They were asked to maintain the same diet the day before each visit and were given a standardised meal to consume the evening before. The standard meal was a supermarket macaroni cheese ready meal. All measurements were taken after a 12-h fast. On each study day, the participants arrived in the morning and provided a fasted capillary blood sample via the finger-prick technique. They then consumed the test drink within $10 \mathrm{~min}$ and provided further capillary blood samples every $15 \mathrm{~min}$ for $2 \mathrm{~h}$. They were asked to rate each drink using a scale of $1-5$ for four drink quality measures: strength, palatability, taste and smell.

\section{Biochemistry}

All blood samples were collected by the finger-prick method using a lancing device and collected into glucose (heparin fluoride) microvette tubes (Sarstedt). Approximately $300 \mathrm{ul}$ of blood was collected at each time point. These were refrigerated and centrifuged at $1509 \boldsymbol{g}$ for $10 \mathrm{~min}$, and the plasma was transferred into serum microvette tubes. Plasma glucose concentrations were measured immediately using the YSI 2300 
Table 1. Composition of the eight coffee drinks used in this study*

\begin{tabular}{|c|c|c|c|c|c|c|c|}
\hline & Drink number & Decaffeinated granules (g) & Added caffeine (mg) & Total caffeine $(\mathrm{mg})$ & Total CQA (mg) & Glucose (g) & Water (ml) \\
\hline \multirow[t]{4}{*}{ Part A } & 1 & 0 & 0 & 0 & 0 & 50 & 400 \\
\hline & 2 & 2 & 94 & 100 & 47 & 50 & 400 \\
\hline & 3 & 4 & 188 & 200 & 94 & 50 & 400 \\
\hline & 4 & 8 & 376 & 400 & 188 & 50 & 400 \\
\hline \multirow[t]{4}{*}{ Part B } & 5 & 0 & 100 & 100 & 0 & 50 & 400 \\
\hline & 6 & 2 & 94 & 100 & 47 & 50 & 400 \\
\hline & 7 & 4 & 88 & 100 & 94 & 50 & 400 \\
\hline & 8 & 8 & 76 & 100 & 188 & 50 & 400 \\
\hline
\end{tabular}

CQA, caffeoylquinic acid.

* Decaffeinated coffee and caffeine powder were dissolved in $200 \mathrm{ml}$ boiling water with $50 \mathrm{~g}$ glucose powder added; $200 \mathrm{ml}$ of cold water was then added to allow quick consumption.

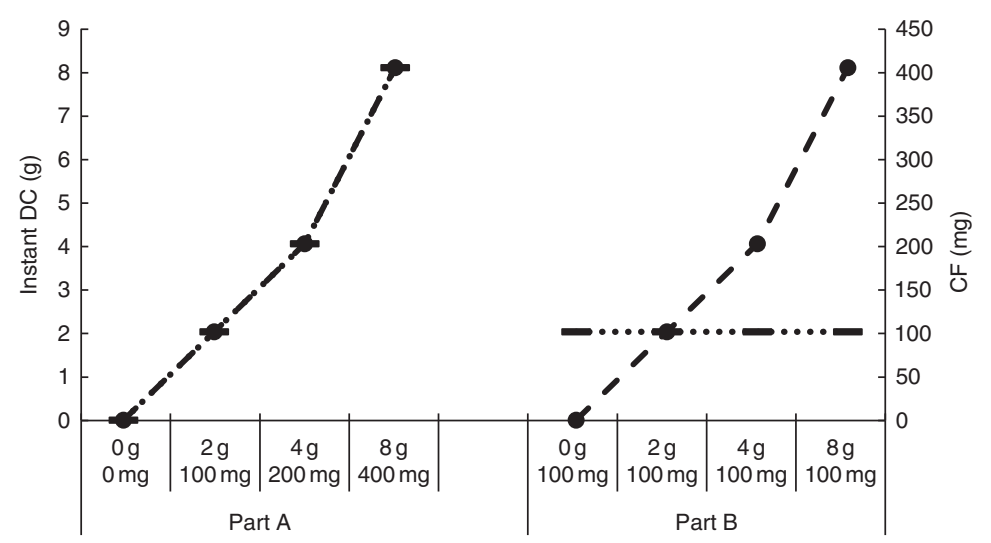

Fig. 1. Diagrammatic representation of the relative proportions of decaffeinated coffee (DC) to caffeine (CF) for the eight test drinks used in this study. In Part $A$, CF rises in proportion to DC, whereas in Part B CF remains constant at $100 \mathrm{mg} .--, \mathrm{DC} ; \ldots-\ldots$, CF.

STAT Plus $^{\mathrm{TM}}$ (YSI Life Sciences). Inter-assay CV were $5 \%$ and intra-assay $\mathrm{CV}$ were $2 \%$ for the glucose analysis. Following glucose analysis, the plasma was frozen at $-20^{\circ} \mathrm{C}$ until the end of the study for batch analysis. Plasma insulin concentrations were measured by colorimetric sandwich ELISA (EMD Millipore). Inter-assay CV were $7 \%$ and intra-assay CV were 3\% for the insulin analysis. The finger-prick technique was used as the blood collected via this method is primarily arterial blood and consequently reflects the absorbed glucose levels more accurately than venous blood. Venous blood glucose values are usually lower than arterial values in the postprandial state ${ }^{(35)}$ as some of the absorbed blood glucose will have been taken up by skeletal muscles before it gets to the sample site.

\section{Calculations and statistical analysis}

Statistical analysis was carried out using IBM SPSS Statistics 19 (IBM). Sample size was set at ten participants, in line with standard practise in glycaemic index testing ${ }^{(36)}$. The incremental AUC (iAUC) for glucose and insulin for each treatment was calculated by the trapezoid method, with the area under baseline being ignored as recommended by the $\mathrm{FAO}^{(37)}$ and as described by Brouns et al. ${ }^{(36)}$. The Matsuda Index $(10,000 /$ $\sqrt{ }([$ fasting glucose $\times$ fasting insulin $] \times$ [mean glucose $\times$ mean insulin during OGTT])) ${ }^{(38)}$ was calculated as a measure of postprandial insulin sensitivity. Overall treatment effects were analysed by repeated measures ANOVA. A Bonferroni adjustment for multiple comparisons was applied when post hoc analysis was used. Statistical significance was taken as $P<0.05$. All results are expressed as mean values with their standard error of the mean, apart from participant characteristics and drink questionnaire results, which are expressed as mean values and standard deviations.

\section{Results}

All the participants were overweight males with a mean age of $30 \cdot 4$ (sD $14 \cdot 2$ ) years, BMI of $27 \cdot 8$ (sD $2 \cdot 2) \mathrm{kg} / \mathrm{m}^{2}$, fasting blood glucose levels of $5.2(\mathrm{sD} \quad 0.5) \mathrm{mmol} / \mathrm{l}$ and were all insulin sensitive (fasting range $5-29 \mathrm{pmol} / \mathrm{l})^{(39)}$. There was no significant difference between visits for fasting glucose and insulin measures. All were regular coffee drinkers with a mean caffeinated coffee intake of $2 \cdot 3$ (sD 1.6) servings/d.

\section{Drink quality}

Mean responses for each of the four drink quality measures strength, palatability, taste and smell - are shown in Table 2. Repeated measures ANOVA tests were performed for Parts A and B. For Part A, an overall significant difference was found for smell ( $P=0.045)$ with no significant differences between individual pairs. No other differences were found in Part A. For Part $\mathrm{B}$, an overall significant difference was found for palatability 
Table 2. Drink questionnaire results*

(Mean values and standard deviations)

\begin{tabular}{|c|c|c|c|c|c|c|c|c|c|}
\hline & \multirow[b]{2}{*}{ Drink number } & \multicolumn{2}{|c|}{ Palatability } & \multicolumn{2}{|c|}{ Strength } & \multicolumn{2}{|c|}{ Taste } & \multicolumn{2}{|c|}{ Smell } \\
\hline & & Mean & SD & Mean & SD & Mean & SD & Mean & SD \\
\hline \multirow[t]{4}{*}{ Part A } & 1 & 2.9 & $1 \cdot 3$ & 2.6 & $1 \cdot 1$ & 2.8 & $1 \cdot 2$ & 2.8 & 0.8 \\
\hline & 2 & 3.9 & 0.6 & $2 \cdot 7$ & 0.7 & 3.6 & 1.0 & 3.2 & 0.6 \\
\hline & 3 & 3.7 & 0.8 & $3 \cdot 3$ & 0.7 & 3.6 & 1.0 & 3.6 & 0.8 \\
\hline & 4 & 3.0 & $1 \cdot 2$ & $3 \cdot 3$ & 0.9 & 2.9 & 1.4 & 3.5 & 0.8 \\
\hline \multirow[t]{4}{*}{ Part B } & 5 & 2.7 & $1 \cdot 1$ & $2 \cdot 1^{a}$ & $1 \cdot 1$ & 2.5 & $1 \cdot 1$ & $2 \cdot 6^{c}$ & 0.8 \\
\hline & 6 & 3.7 & 0.7 & $2 \cdot 5^{a}$ & 0.8 & 3.6 & 0.7 & $3 \cdot 7^{d}$ & 0.5 \\
\hline & 7 & $4 \cdot 1$ & 0.6 & $3 \cdot 1$ & 0.7 & $3 \cdot 8$ & 0.8 & 3.7 & 0.8 \\
\hline & 8 & 3.8 & 0.8 & $3 \cdot 6^{b}$ & 0.8 & 3.4 & 0.8 & 3.5 & 0.8 \\
\hline
\end{tabular}

a,b,c.d Mean values within a column/part with unlike superscript letters were significantly different $(P<0.05)$.

* Participants rated the control and coffee drinks on a scale of 1-5 for palatability, strength, taste and smell.

(a)

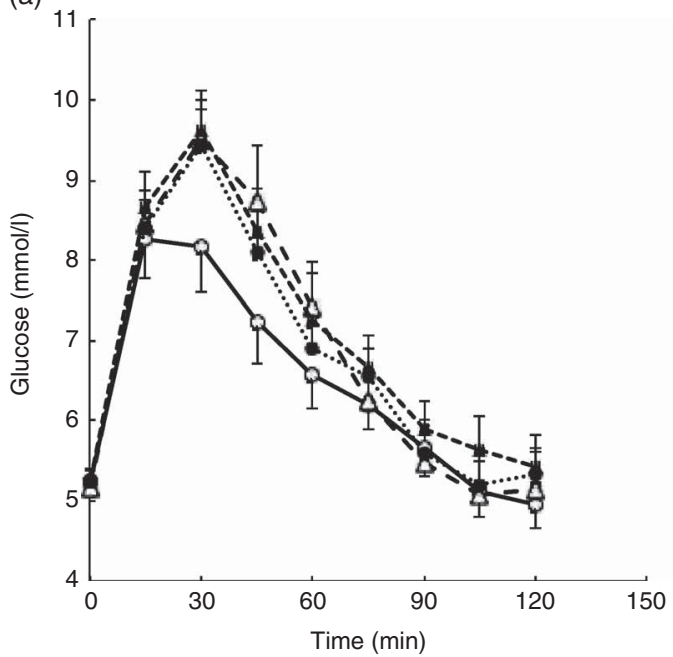

(b)

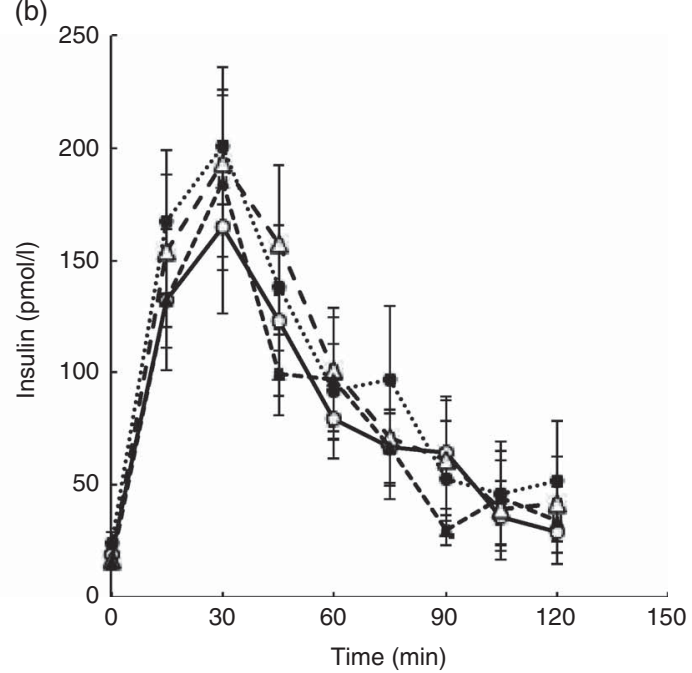

Fig. 2. Postprandial glucose (a) and insulin (b) responses for a control drink (water) $v$. three different doses of decaffeinated coffee (DC) with increasing amounts of caffeine (CF) added, equivalent to 1, 2 and 4 servings of standard caffeinated coffee. All four drinks also contained $50 \mathrm{~g}$ glucose. Error bars are standard error of the mean $(n 10)$. A significant difference between treatments was observed for glucose (repeated measures ANOVA on time point data, $P=0.008$ ). $-0-, 0 \mathrm{~g} \mathrm{DC}, 0 \mathrm{mg}$ CF; $-\Delta-, 2$ g DC, $100 \mathrm{mg} \mathrm{CF} ;-\_-\cdot, 4 \mathrm{~g} \mathrm{DC}, 200 \mathrm{mg} \mathrm{CF} ; \cdots \bullet \cdot, 8 \mathrm{~g} \mathrm{DC}, 400 \mathrm{mg} \mathrm{CF}$.

$(P=0.020)$ and taste $(P=0.036)$ with no significant differences between individual pairs. A significant difference in strength $(P<0.001)$ was detected in Part B, with the post boc analysis revealing drink 8 to have been perceived as stronger than both drinks $6(P=0.040)$ and $5(P=0.002)$. A difference in smell $(P=0.025)$ was also found in Part B, with the post hoc analysis revealing a difference between drinks 5 and $6(P=0 \cdot 001)$.

\section{Glucose and insulin}

For Part A, where the amount of caffeine was varied in line with the DC dose, there was a significant difference between treatments for glucose. Repeated measures ANOVA on the glucose time points showed a significant treatment effect $(P=0.008)$ and a time $\times$ treatment effect $(P=0.022)$. Post hoc analysis showed a significant difference between the control drink (drink 1 in Table 1 ) and both the one- and two-serving equivalents (drinks 2 and 3$)(P \leq 0.032)$. A significant difference was also found for both iAUC $(P=0.019)$ and peak values $(P=0.006)$, with the post hoc analysis showing a significant difference between drinks 1 and 2 for both iAUC $(P=0.008)$ and peak values $(P=0.006)$ and a trend for a difference between drinks 1 and 3 for peak value $(P=0.056)$. The mean peak blood glucose concentration for the control (drink 1) was $8.25 \mathrm{mmol} / \mathrm{l}$, whereas the peak for Part A was $9.62 \mathrm{mmol} / \mathrm{l}$ (for the two servings equivalent: drink 3), a mean increase of $17 \%(1.37 \mathrm{mmol} / \mathrm{l})$ in peak values. No other differences between treatments were detected. There was no difference between treatments for postprandial insulin or insulin sensitivity as measured by the Matsuda Index. The 2-h postprandial glucose and insulin responses for Part A are shown in Fig. 2. The mean iAUC and peak values for glucose and insulin for Parts A and B are shown in Table 3.

For Part B of the study, where all drinks contained $100 \mathrm{mg}$ caffeine, a significant difference in glucose was found for peak values only $(P=0.049)$, with no difference between any 
Table 3. Mean incremental AUC (iAUC) and peak glucose and insulin values for the 2-h postprandial period (Mean values and standard deviations)

\begin{tabular}{|c|c|c|c|c|c|c|c|c|c|c|}
\hline & \multirow[b]{2}{*}{ Drink number } & \multirow[b]{2}{*}{ Decaffeinated granules $(\mathrm{g}) /$ total caffeine $(\mathrm{mg})$} & \multicolumn{2}{|c|}{ Glucose iAUC } & \multicolumn{2}{|c|}{ Peak glucose $(\mathrm{mmol} / \mathrm{l})$} & \multicolumn{2}{|c|}{ Insulin iAUC } & \multicolumn{2}{|c|}{ Peak insulin (pmol/l) } \\
\hline & & & Mean & SD & Mean & SD & Mean & SD & Mean & SD \\
\hline \multirow[t]{4}{*}{ Part A } & 1 & 0/0 & $172^{\mathrm{a}}$ & 89 & $8 \cdot 9^{a}$ & 1.3 & 8238 & 6114 & 193 & 109 \\
\hline & 2 & $2 / 100$ & $237^{b}$ & 102 & $10 \cdot 0^{b}$ & 1.4 & 10294 & 7376 & 239 & 139 \\
\hline & 3 & $4 / 200$ & 243 & 108 & 9.8 & 1.3 & 8521 & 5141 & 206 & 111 \\
\hline & 4 & $8 / 400$ & 218 & 117 & 9.5 & 1.4 & 10098 & 7848 & 224 & 97 \\
\hline \multirow[t]{4}{*}{ Part B } & 5 & $0 / 100$ & $210^{\mathrm{b}, \mathrm{c}}$ & 77 & 8.9 & 1.3 & 6094 & 4374 & 151 & 74 \\
\hline & 6 & $2 / 100$ & 237 & 104 & 9.8 & 1.5 & 6887 & 4523 & 159 & 61 \\
\hline & 7 & $4 / 100$ & 224 & 110 & 9.6 & 1.3 & 7198 & 5263 & 158 & 72 \\
\hline & 8 & $8 / 100$ & 207 & 110 & 9.3 & 1.1 & 7584 & 5845 & 205 & 84 \\
\hline
\end{tabular}

a,b,c Mean values within a column with unlike superscript letters were significantly different $(P<0.05)$.

(a)

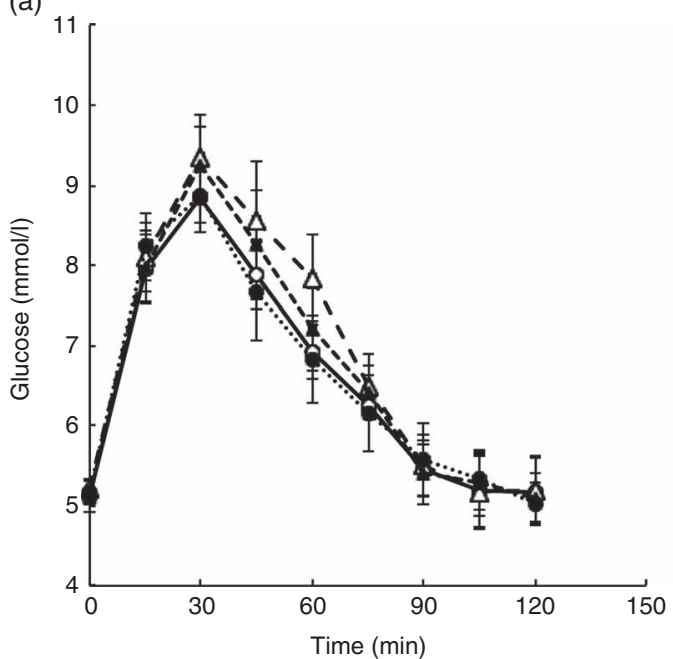

(b)

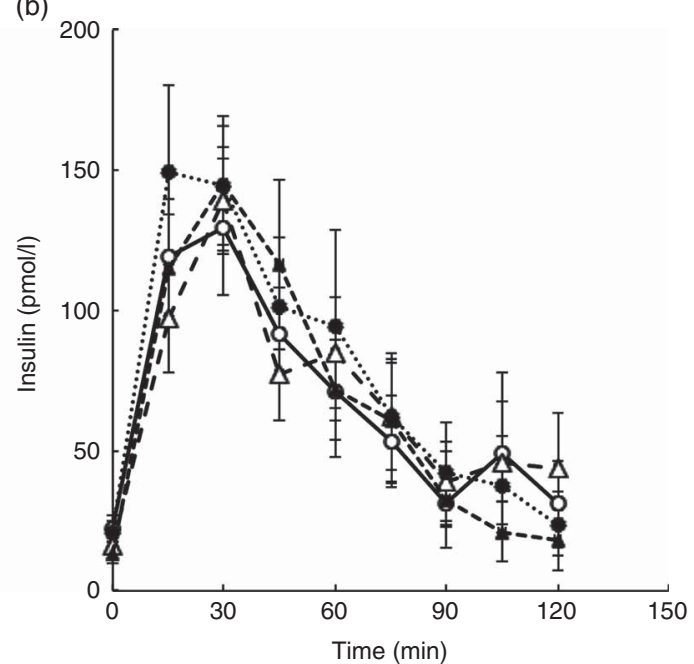

Fig. 3. Postprandial glucose (a) and insulin (b) responses for a control drink (water) $v$. three different doses of decaffeinated coffee (DC) with 100 mg caffeine (CF) added to each. All four drinks also contained $50 \mathrm{~g}$ glucose. Error bars are standard error of the mean ( $n$ 10). No significant difference between treatments was observed for either glucose or insulin (repeated measures ANOVA on time point data). ———, $0 \mathrm{~g} \mathrm{DC,} 100 \mathrm{mg} \mathrm{CF} ;-\Delta-$, $2 \mathrm{~g} \mathrm{DC}, 100 \mathrm{mg} \mathrm{CF} ;-\rightarrow-\cdot, 4 \mathrm{~g} \mathrm{DC}, 100 \mathrm{mg}$ CF; -.., $8 \mathrm{~g} \mathrm{DC}, 100 \mathrm{mg} \mathrm{CF}$.

individual pairs in the post boc analysis. There were no significant differences for insulin responses or insulin sensitivity between treatments, although there was a time $\times$ treatment effect for insulin $(P=0.013)$. The 2 -h postprandial glucose and insulin responses for Part B are shown in Fig. 3.

A paired $t$ test comparing the glucose iAUC for the two control drinks (1 and 5) showed a significant difference between the two $(P=0.045)$, with the control drink containing caffeine (drink 5) having a $22 \%$ higher mean iAUC. There were no significant differences between the two control drinks for any other measures.

\section{Discussion}

Our study adds to the body of evidence from previous acute studies demonstrating a temporary worsening in glucose response following coffee ingestion. We have shown, for the first time, that a single serving of instant caffeinated coffee, as typically consumed in the UK, is sufficient to disrupt the 2-h postprandial glucose response. Furthermore, Part B of our study demonstrates that the amount of caffeine found in one serving of coffee can attenuate any possible beneficial effects of escalating doses of the other coffee components.

Gavrieli et al. ${ }^{(40)}$ used two different amounts of caffeinated coffee, 3 and $6 \mathrm{mg} / \mathrm{kg}$ body weight (mean: 228 and $457 \mathrm{mg}$ ), which were similar to the two higher amounts of caffeine used in Part A (200 and $400 \mathrm{mg}$, drinks 3 and 4) and found similar results. In line with our study, they reported a higher glucose iAUC in both coffee doses compared with control, with no significant dose-response effect. Although they did not find any overall intervention effect for the glucose response, they did find a time $\times$ treatment effect. Our post hoc analysis found the increased glucose response only in the two lower doses of coffee plus caffeine (drinks 2 and 3) and not in the highest dose. It is unlikely that a greater insulin response to the high caffeine levels had suppressed the glucose response in this case, as we found no significant differences between drinks for the insulin response. Furthermore, although Beaudoin et $a l^{(41)}$ did find a dose-response effect on insulin, they did not find a direct suppression of glucose as a result. We also observed a difference between treatments in the timing of the peak glucose values, with 
the control drink peaking at 15 min while all the others peaked later at $30 \mathrm{~min}$. This is consistent with the theory that coffee may exert a beneficial effect by delaying glucose absorption ${ }^{(24,25)}$.

In Part B of our study, we found no overall treatment effect even at the highest dose of DC ( $8 \mathrm{~g})$, suggesting that even a relatively small dose of caffeine $(100 \mathrm{mg}$, equivalent to that found in one serving of caffeinated coffee) may be enough to override any potential beneficial effects of increasing other coffee compounds. We did see a difference in peak glucose values across the four treatments in Part B; however, the study was not powered to detect pair-wise difference in individual treatments. The control drink in Part B also contained $100 \mathrm{mg}$ caffeine, unlike in Part A where it was simply water plus glucose, which would explain why there was no significant difference between the control drink and any of the coffee drinks. When the two control drinks were compared, we found the iAUC to be significantly higher for the caffeine-containing control (drink 5), suggesting that it is indeed caffeine that is causing the increased glycaemic response in Part A. It should be noted that the CGA content of coffees can vary considerably, depending on bean composition, processing and brewing method $^{(29)}$, and a detailed analysis of our coffee samples found it to have a relatively low total CQA content of $23.5 \mathrm{mg} / \mathrm{g}$ (CQA being the largest proportion of CGA in coffee). This is equivalent to approximately $47 \mathrm{mg}$ in our lowest dose coffee and $188 \mathrm{mg}$ in our highest dose (Table 1). This is low compared with espresso-type coffees, which have a mean CQA content of $145 \mathrm{mg} /$ serving (range $24-422 \mathrm{mg})^{(29)}$; however, it is comparable to the mean CQA content of six brands of instant DC, previously measured at $24.4 \mathrm{mg} / \mathrm{g}^{(24)}$. Nevertheless, these relatively low levels in our coffee may partially explain why we saw no effect of escalating doses of CGA in Part B of our study.

We did not demonstrate any dose-response effect of coffee on blood glucose or insulin levels, either when the caffeine was increased along with the coffee (Part A) or when the caffeine was kept constant (Part B). This is in contrast with a recent study that found a dose-response effect when using caffeine alone ${ }^{(41)}$; however, the caffeine-only study had more participants ( $n$ 24), and - as noted previously - caffeine has been shown to produce a greater effect than coffee on postprandial glucose and insulin levels. The lack of a dose-response effect may also be due to the high inter- and intra-individual variations in glycaemic response, which was apparent when individuals' responses were examined and is in line with that previously demonstrated ${ }^{(42)}$. Our relatively low number of participants (ten), although being the recommended minimum number for GI testing, may not be sufficient to determine a dose-response relationship. A post hoc power calculation revealed our study to be underpowered to detect a difference in insulin levels. In order for the detected differences in insulin to be statistically significant at $80 \%$ power, we would have needed more than twenty-three participants. This is based on the result that found 5/10 subjects had an increase in insulin levels after coffee intake compared with control and 5/10 subjects had a decrease in insulin levels, and thus no consistent pattern in response was observed. The doses given to our participants were not adjusted according to their body weight in contrast with the previously discussed studies ${ }^{(20-22,40,41)}$ as we wanted to reflect normal nutritional intake patterns. When converted to milligram caffeine per kilogram body weight, our lowest dose (100 mg caffeine) gave a mean value of $1.2 \mathrm{mg} / \mathrm{kg}$ (range $0.9-1.5 \mathrm{mg} / \mathrm{kg}$ ) and our highest dose (400 mg caffeine) was $4.6 \mathrm{mg} / \mathrm{kg}$ (range $3.7-6.1 \mathrm{mg} / \mathrm{kg}$ ). This variation in dose between participants may be a confounder in determining a dose-response relationship; however, it does not detract from the main result, which is that a single cup of coffee as drunk in the UK increases the postprandial glycaemic response. Another possible confounder may be different genetic polymorphisms in the CYP1A2 gene that have been shown to affect the rate of caffeine metabolism in humans ${ }^{(43)}$. Unfortunately, our participants were not genotyped for these polymorphisms as this may have partly explained the high inter-individual variations we found. An obvious difference between the control and coffee drinks is the presence/absence of coffee with its characteristic bitter taste. The hedonic properties of food/drink have been shown to impact acutely on postprandial nutrient handling ${ }^{(44)}$, and thus it was important to exclude this as a simple pre-ingestive mechanism. Interestingly, the only drink quality difference from Part A (where a postprandial glucose difference was found) was with 'smell'; there was no overall impact on palatability. This would indicate that the impact of coffee on glucose metabolism was due to a true post-ingestive mechanism. In light of our results, and those of others discussed previously, it would be advisable for establishments carrying out GI testing not to offer coffee as an accompaniment to the test food.

In conclusion, we have demonstrated for the first time that a single serving of coffee significantly increases the postprandial glycaemic response in healthy overweight men. What is not known is whether this statistically significant increase in blood glucose is physiologically relevant and the implications for individuals with abnormal glucose tolerance. Although we observed a high percentage difference in peak values, it was quickly resolved in insulin-sensitive individuals with all treatments, including control, displaying similar responses from $60 \mathrm{~min}$ onwards. It is important that further investigations are carried out in those with impaired fasting glycaemia and T2DM where the effects of coffee may be clinically relevant. It should be noted that this was an acute study and its results cannot be translated simply into chronic effects. Longer-term interventions investigating these chronic effects are now needed if we are to confirm the beneficial effects of coffee suggested by the epidemiology.

\section{Acknowledgements}

The authors thank Professor Alan Crozier and Dr Iziar Amaia Ludwig Sanz Orrio (University of Glasgow) for providing the detailed coffee analysis and Dr Caroline Bodinham, Dr Fariba Shojaee-Moradie and Dr Shelagh Hampton (University of Surrey) for guidance with laboratory techniques.

This work was supported by the Biotechnology and Biological Sciences Research Council (BBSRC) and Campden BRI as part of a BBSRC CASE Studentship.

T. M. R., M. D. R. and M. N. C. designed the research; T. M. R. conducted the research; T. M. R. analysed the data; T. M. R. and M. D. R. wrote the paper. All the authors read and approved the final version of the manuscript.

T. M. R., M. N. C., S. P., G. C. and M. D. R. have no conflicts of interest. 


\section{References}

1. World Health Organization (2013) Fact sheet 312 diabetes. http://www.who.int/mediacentre/factsheets/fs312/en/ (accessed December 2014).

2. Odegaard AO, Pereira MA, Koh W-P, et al. (2008) Coffee, tea, and incident type 2 diabetes: the Singapore Chinese Health Study. Am J Clin Nutr 88, 979-985.

3. Bhupathiraju SN, Pan A, Malik VS, et al. (2013) Caffeinated and caffeine-free beverages and risk of type 2 diabetes. Am J Clin Nutr 97, 155-166.

4. Rosengren A, Dotevall A, Wilhelmsen L, et al. (2004) Coffee and incidence of diabetes in Swedish women: a prospective 18-year follow-up study. J Intern Med 255, 89-95.

5. Ding M, Bhupathiraju SN, Chen M, et al. (2014) Caffeinated and decaffeinated coffee consumption and risk of type 2 diabetes: a systematic review and a dose-response metaanalysis. Diabetes Care 37, 569-586.

6. Salazar-Martinez E, Willett WC, Ascherio A, et al. (2004) Coffee consumption and risk for type 2 diabetes mellitus. Ann Intern Med 140, 1-8.

7. Van Dam RM, Willett WC, Manson JE, et al. (2006) Coffee, caffeine, and risk of type 2 diabetes: a prospective cohort study in younger and middle-aged U.S. women. Diabetes Care 29, 398-403.

8. Van Dam RM \& Feskens EJM (2002) Coffee consumption and risk of type 2 diabetes mellitus. Lancet 360, 1477-1478.

9. Tuomilehto J, Hu G, Bidel S, et al. (2004) Coffee consumption and risk of type 2 diabetes mellitus among middle-aged Finnish men and women. JAMA 291, 1213-1219.

10. Dulloo A, Geissler C, Horton T, et al. (1989) Normal caffeine consumption: influence on thermogenesis and daily energy expenditure in lean and postobese human volunteers. Am J Clin Nutr 49, 44-50.

11. Koot P \& Deurenberg P (1995) Comparison of changes in energy expenditure and body temperatures after caffeine consumption. Ann Nutr Metab 39, 135-142.

12. Bakuradze T, Boehm N, Janzowski C, et al. (2011) Antioxidant-rich coffee reduces DNA damage, elevates glutathione status and contributes to weight control: results from an intervention study. Mol Nutr Food Res 55 , 793-797.

13. Gavrieli A, Karfopoulou E, Kardatou E, et al. (2013) Effect of different amounts of coffee on dietary intake and appetite of normal-weight and overweight/obese individuals. Obesity (Silver Spring) 21, 1127-1132.

14. Kempf K, Kolb H, Gärtner B, et al. (2014) Cardiometabolic effects of two coffee blends differing in content for major constituents in overweight adults: a randomized controlled trial. Eur J Nutr 54, 845-854.

15. Ohnaka K, Ikeda M, Maki T, et al. (2012) Effects of 16-week consumption of caffeinated and decaffeinated instant coffee on glucose metabolism in a randomized controlled trial. J Nutr Metab 2012, article ID 207426.

16. Wedick NM, Brennan AM, Sun Q, et al. (2011) Effects of caffeinated and decaffeinated coffee on biological risk factors for type 2 diabetes: a randomized controlled trial. Nutr J 10, 93.

17. Yatagai T, Nagasaka S, Taniguchi A, et al. (2003) Hypoadiponectinemia is associated with visceral fat accumulation and insulin resistance in Japanese men with type 2 diabetes mellitus. Metabolism 52, 1274-1278.

18. Robinson LE, Savani S, Battram DS, et al. (2004) Caffeine ingestion before an oral glucose tolerance test impairs blood glucose management in men with type 2 diabetes. J Nutr 134, $2528-2533$.
19. Greenberg JA, Owen DR \& Geliebter A (2010) Decaffeinated coffee and glucose metabolism in young men. Diabetes Care 33, 278-280.

20. Beaudoin M-S, Robinson LE \& Graham TE (2011) An oral lipid challenge and acute intake of caffeinated coffee additively decrease glucose tolerance in healthy men. $J$ Nutr 141, 574-581.

21. Moisey LL, Kacker S, Bickerton AC, et al. (2008) Caffeinated coffee consumption impairs blood glucose homeostasis in response to high and low glycemic index meals in healthy men. Am J Clin Nutr 87, 1254-1261.

22. Krebs JD, Parry-Strong A, Weatherall M, et al. (2012) A crossover study of the acute effects of espresso coffee on glucose tolerance and insulin sensitivity in people with type 2 diabetes mellitus. Metabolism 61, 1231-1237.

23. Battram DS, Arthur R, Weekes A, et al. (2006) The glucose intolerance induced by caffeinated coffee ingestion is less pronounced than that due to alkaloid caffeine in men. J Nutr 136, 1276-1280.

24. Johnston KL, Clifford MN \& Morgan LM (2003) Coffee acutely modifies gastrointestinal hormone secretion and glucose tolerance in humans: glycemic effects of chlorogenic acid and caffeine. Am J Clin Nutr 78, 728-733.

25. Welsch CA, Lachance PA \& Wasserman BP (1989) Dietary phenolic compounds: inhibition of $\mathrm{Na}^{+}$-dependent D-glucose uptake in rat intestinal brush border membrane vesicles. J Nutr 119, 1698-1704.

26. Shearer J, Farah A, de Paulis T, et al. (2003) Quinides of roasted coffee enhance insulin action in conscious rats. $J$ Nutr 133, 3529-3532.

27. Van Dijk AE, Olthof MR, Meeuse JC, et al. (2009) Acute effects of decaffeinated coffee and the major coffee components chlorogenic acid and trigonelline on glucose tolerance. Diabetes Care 32, 1023-1025.

28. Thom E (2007) The effect of chlorogenic acid enriched coffee on glucose absorption in healthy volunteers and its effect on body mass when used long-term in overweight and obese people. J Int Med Res 35, 900-908.

29. Crozier TWM, Stalmach A, Lean MEJ, et al. (2012) Espresso coffees, caffeine and chlorogenic acid intake: potential health implications. Food Funct 3, 30-33.

30. Gavrieli A, Yannakoulia M, Fragopoulou E, et al. (2011) Caffeinated coffee does not acutely affect energy intake, appetite, or inflammation but prevents serum cortisol concentrations from falling in healthy men. J Nutr $\mathbf{1 4 1}$, 703-707.

31. Gilbert RM, Marshman JA, Schwieder M, et al. (1976) Caffeine content of beverages as consumed. Can Med Assoc J 114, 205-208.

32. The British Coffee Association (2015) Coffee facts. http:// www.britishcoffeeassociation.org/about_coffee/coffee_facts/ (accessed February 2015).

33. Ludwig IA, Sanchez L, Caemmerer B, et al. (2012) Extraction of coffee antioxidants: impact of brewing time and method. Food Res Int 48, 57-64.

34. McCusker RR, Goldberger BA \& Cone EJ (2003) Caffeine content of specialty coffees. I Anal Toxicol 27, 520-522.

35. Larsson-Cohn U (1976) Differences between capillary and venous blood glucose during oral glucose tolerance tests. Scand J Clin Lab Invest 36, 805-808.

36. Brouns F, Bjorck I, Frayn KN, et al. (2005) Glycaemic index methodology. Nutr Res Rev 18, 145-171.

37. World Health Organization (1998) Carbohydrates in human nutrition. Report of a Joint FAO/WHO Expert Consultation. FAO Food Nutr Pap 66, 1-140. 
38. Matsuda M \& DeFronzo RA (1999) Insulin sensitivity indices obtained from oral glucose tolerance testing: comparison with the euglycemic insulin clamp. Diabetes Care 22, 1462-1470.

39. Strazzullo P, Barbato A, Siani A, et al. (2008) Diagnostic criteria for metabolic syndrome: a comparative analysis in an unselected sample of adult male population. Metabolism 57, 355-361.

40. Gavrieli A, Fragopoulou E, Mantzoros CS, et al. (2013) Gender and body mass index modify the effect of increasing amounts of caffeinated coffee on postprandial glucose and insulin concentrations; a randomized, controlled, clinical trial. Metabolism 62, 1099-1106.
41. Beaudoin M-S, Allen B, Mazzetti G, et al. (2013) Caffeine ingestion impairs insulin sensitivity in a dose-dependent manner in both men and women. Appl Physiol Nutr Metab 38, 140-147.

42. Vega-López S, Ausman LM, Griffith JL, et al. (2007) Interindividual variability and intra-individual reproducibility of glycemic index values for commercial white bread. Diabetes Care 30, 1412-1417.

43. Yang A, Palmer AA \& de Wit H (2010) Genetics of caffeine consumption and responses to caffeine. Psychopharmacology (Berl) 211, 245-257.

44. Robertson MD, Jackson KG, Williams CM, et al. (2001) Prolonged effects of modified sham feeding on energy substrate mobilization. Am J Clin Nutr 73, 111-117. 